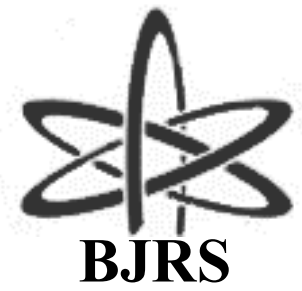

BRAZILIAN JOURNAL

$\mathrm{OF}$

RADIATION SCIENCES

08-01 (2020) 01-10

\title{
Avaliação da exposição do público e da tripulação de bordo em voos comerciais domésticos do Brasil
}

\author{
Rochedo, E.R.R. ${ }^{a}$, Alves, V.A. ${ }^{\mathrm{b}}$, Wasserman, M.A.V. ${ }^{\mathrm{a}}$ \\ ${ }^{a}$ Instituto de Radioproteção e Dosimetria/Departamento de Radioproteção Ambiental, CEP 22793-127, Rio de Janeiro, \\ RJ, Brasil \\ ${ }^{b}$ Instituto Militar de Engenharia/Departamento de Engenharia Nuclear, CEP 22290-270, Rio de Janeiro, RJ, Brasil \\ elainerochedo@gmail.com
}

\section{RESUMO}

A exposição à radiação cósmica em viagens de avião é significativamente maior do que a exposição ao nível do solo e varia de acordo com a rota, a altitude e o tempo de voo. O programa CARI-6, desenvolvido pela Administração Federal de Aviação dos EUA, efetua o cálculo da dose efetiva de radiação cósmica galáctica recebida por um indivíduo em um avião a voar a rota mais curta entre dois aeroportos do mundo. $O$ objetivo deste projeto é o de estimar a contribuição de exposição à radiação cósmica em voos comerciais domésticos para a população brasileira. Um banco de dados, incluindo cerca de 4.000 voos domésticos no Brasil, foi implementado em planilhas do Excel com base em informações de voos para novembro de 2011. O banco de dados descreve a origem e destino de cada voo, hora de partida e chegada, o tipo de avião, número de passageiros, as horas de voo (incluindo períodos de decolagem, pouso e altitude de cruzeiro) e número de voos por ano. Doses individuais para voos domésticos no Brasil variaram de 1,8 a 8,8 $\mu \mathrm{Sv}$. Considerando-se o limite ocupacional de 850 h de voo por ano para tripulantes e números de voos de cada rota, a dose ocupacional média seria de cerca de 0,76 mSv/a. Doses coletivas, para o número total de voos por ano foram estimadas em 429 e 22 pessoa.Sv/a para os passageiros e membros da tripulação, respectivamente.

Keywords: Radiação cósmica; voos comerciais domésticos; exposição do público; exposição da tripulação 


\begin{abstract}
The exposure to cosmic radiation in aircraft travel is significantly higher than that at ground level and varies with the route, the altitude of flight, and the flight time. The computer program CARI-6, developed by the U.S. Federal Aviation Administration, calculates the effective dose of galactic cosmic radiation received by an individual in an aircraft flying the shortest route between two airports of the world. The aim of this project is to estimate the contribution of cosmic radiation exposure on domestic commercial flights to the Brazilian population. A database, including about 4,000 domestic flights in Brazil, was implemented in Excel spreadsheets based on data flights information for November 2011. Main fields included on the database are the origin and destination of flights, time of departure and arrival, plane type, number of passengers, flight times (including periods of take-off, landing and cruse altitude times) and number of flights per year. Doses for domestic flights in Brazil ranged from 1.8 to $8.8 \mu \mathrm{Sv}$. Considering the occupational limit of $850 \mathrm{~h}$ of flight per year for crewmembers and numbers of flights for each route, average occupational dose would be about $0.76 \mathrm{mSv} / \mathrm{y}$. Collective doses, for the total number of flights per year and airplane types were estimated to be 429 and 22 manSv/y for passengers and crewmembers, respectively.
\end{abstract}

Key words: Cosmic radiation, Domestic commercial flights, public exposure, crew expsore 


\section{INTRODUÇÃO}

A radiação sempre esteve presente na biosfera, onde a vida se desenvolveu. A maior contribuição, para as várias exposições à radiação recebidas pelo homem, vem de fontes naturais. Uma característica da irradiação natural é que ela vem sendo recebida por toda a população do mundo, por um longo período de tempo, a uma taxa relativamente constante. De um modo geral, a exposição do homem a fontes naturais não modificadas, isto é, que não sofreram ação antropogênica, é uma condição inevitável da vida na Terra e não está sujeita ao controle regulatório. As principais fontes de radiação natural, que contribuem para a exposição humana são a radiação cósmica e a radiação terrestre [1].

Toda a população do mundo está exposta à radioatividade natural. As principais fontes de radiação natural que contribuem para a exposição do homem são:

(a) Radiação cósmica e os radionuclídeos cosmogênicos, formados pela interação da radiação cósmica com o ar; e,

(b) Fontes terrestre de radiação, que são os radionuclídeos de vida longa que existem na Terra desde a sua formação, bilhões de anos atrás, e que ainda não decaíram substancialmente, e seus descendentes [1].

A maior parte da radiação cósmica que atinge a Terra é originada fora do sistema solar e alcança a atmosfera a uma taxa razoavelmente constante. A radiação cósmica sofre interações ao longo de seu caminho na atmosfera até alcançar a superfície da Terra, produzindo o que se denomina radiação cósmica secundária. Nestas interações, perde energia e dá origem a várias partículas e aos radionuclídeos cosmogênicos.

A rigidez de uma determinada radiação é definida pela relação entre seu momento e sua carga. Cada latitude geomagnética pode ser caracterizada por uma rigidez de corte tal que partículas com rigidez inferior a este valor não podem penetrar na atmosfera nesta latitude. As latitudes equatoriais são as mais protegidas. Outro fator importante na exposição à radiação cósmica são os ciclos solares. Manchas solares aparecem quando campos magnéticos intensos emergem da superfície do Sol. Como a transferência de energia é bloqueada pelos campos magnéticos, as manchas solares são mais frias do que as áreas circunvizinhas e parecem escuras ao observador. Os raios cósmicos 
galácticos são observados na Terra em proporção inversa à da fase do ciclo de manchas solares, isto é, o período de menor intensidade de radiação cósmica na superfície da Terra corresponde a um período de maior intensidade de eventos solares.

A exposição aos raios cósmicos também varia com outros fatores, além do efeito de latitude descrito. Como o ar age como um absorvedor de raios cósmicos secundários, um efeito de altitude é então esperado. A variação dos componentes ao longo de sua trajetória afeta a taxa de dose causada pelos raios cósmicos secundários na atmosfera. Ao nível do solo, o componente de múons é responsável pela maior contribuição para a dose. Em altitudes de voos comerciais, nêutrons, elétrons, pósitrons, fótons e prótons são os componentes mais significativos. Em maiores altitudes, o componente de núcleos pesados também deve ser considerado [1].

A média mundial estimada pelo UNSCEAR - Comitê Científico das Nações Unidas para os Efeitos da Radiação Atômica para a dose efetiva devido à radiação cósmica ao nível do solo é de $0,38 \mathrm{mSv}$ por ano, cerca de $16 \%$ da dose média mundial a partir de fontes naturais [1].

A exposição à radiação cósmica, em viagem de avião é significativamente mais elevada do que aquela recebida ao nível do mar e varia de acordo com o percurso, devido ao efeito de latitude, a altitude de voo, o tempo de voo, e o ano, devido ao efeito do ciclo solar em fluxo de raios cósmicos galácticos. Em relação ao público em geral, esta exposição não está sujeita a regulamentação, porque ela é considerada como uma atividade voluntária, embora os voos comerciais representem um aumento da exposição tecnológica de pessoas à radiação natural.

O objetivo deste trabalho foi estimar a contribuição de exposição à radiação cósmica em voos comerciais para a população brasileira, incluindo a exposição dos tripulantes de voo e as doses de viajantes frequentes nas principais rotas nacionais. $\mathrm{O}$ trabalho deve servir como base para futuras comparações de riscos decorrentes do crescimento da aviação civil no país e para acessar a contribuição desta fonte à exposição total do público no país. 


\section{METODOS}

Em uma primeira etapa foram selecionados voos padrão para executar as simulações utilizando o código CARI-6, a fim de fazer uma análise de sensibilidade inicial sobre os principais parâmetros, tais como tempo de voo, altitude de voo, latitude, tempos de decolagem e aterrissagem [2]. De acordo com os resultados dessa análise, utilizando os dados fornecidos pela revista Pan Rotas [3] um banco de dados foi implementado em planilhas do Excel contendo todos os voos nacionais no mês de novembro de 2011 [4].

Para o presente trabalho, foram considerados apenas os voos domésticos. Os campos descritos no banco de dados são a origem e destino dos voos; tempo de partida e chegada; frequência semanal de cada voo; tipo de avião; número de passageiros (capacidade total para o tipo de avião); companhia aérea e número do voo; tempo total de voo; Sigla ICAO (International Civil Aviation Organization) para os aeroportos de origem e destino do voo; e, altitude média de cruzeiro e período de tempo utilizado da decolagem até atingir a altitude de cruzeiro e tempo utilizado desde a altitude de cruzeiro até a aterrissagem do avião. O banco de dados final inclui 3872 voos domésticos, com mais de 1.000.000 de voos individuais por ano no Brasil.

Em seguida, o banco de dados foi complementado com dados sobre a tripulação aérea. O número de membros da tripulação foi estimado em função do tipo de avião, com base na informação fornecida pela Boeing [5], complementado por características dos aviões disponibilizadas na internet por seus construtores.

Informações sobre horários de decolagem e de aterragem e sobre as altitudes de cruzeiro foram recolhidas a partir de consultas com especialistas e dados de companhias aéreas oficiais (DECEA Departamento de Controle do Espaço Aéreo - informações pessoais; Coordenador do tráfego aéreo do Aeroporto de Jacarepaguá, no Rio de Janeiro, William Macedo de Lima, informação pessoal).

Doses de voos individuais foram avaliados utilizando o programa de computador CARI-6, desenvolvido por os Administração Federal de Aviação dos Estados Unidos [2]. Este programa calcula a dose efetiva de radiação cósmica galáctica recebida por um indivíduo em um avião a voar a rota mais curta entre dois aeroportos do mundo. O programa leva em conta as mudanças de altitude e latitude durante o curso de um voo, os efeitos de decolagem e de aterrissagem, e o efeito do ciclo solar, a partir dos dados do perfil de voo digitado pelo usuário. O programa foi utilizado 
para estimar a exposição dos tripulantes e o mesmo valor foi aplicado a membros adultos do público.

\section{RESULTADOS E DISCUSSÕES}

As doses médias avaliadas para voos domésticos individuais dentro do Brasil, calculado pelo software CARI-6 para o ano de 2011, variou de 0,3 a $8,8 \mu \mathrm{Sv}$.

Com a taxa de ocupação média de 73\% observada para os voos domésticos em 2012 no Brasil [6], estima-se que cerca de 100 milhões de pessoas voam anualmente em voos domésticos no Brasil, com uma dose coletiva total de cerca de 421 pessoa.Sv por ano. Um resumo dos resultados com base no estado de origem dos voos é apresentado na Tabela 1.

Mais de $40 \%$ de todos os passageiros voam a partir de três cidades: São Paulo (20,1\%), Rio de Janeiro (12,5\%) e Brasília (8,9\%). A contribuição para a dose coletiva total, é de $21 \%$ para voos com partida e chegada a São Paulo, 12\% para o Rio de Janeiro, e 11\% para Brasília. Para os voos comerciais considerados neste estudo, com base na listagem da Pan Rotas [3], Rio de Janeiro e São Paulo cidades têm voos a partir de dois aeroportos cada um e Brasília tem voos relacionados a apenas um aeroporto.

A avaliação da dose realizada para voos diários mais usuais no país, que são aquelas entre Rio de Janeiro e São Paulo, apresenta uma dose média de $1,8 \mu \mathrm{Sv}$ (desvio padrão de 0,43 $\mu \mathrm{Sv}$ ); doses médias de voos para Brasília a partir do Rio de Janeiro e de São Paulo são 3,37 $\mu \mathrm{Sv}$ e 3,24 $\mu \mathrm{Sv}$, respectivamente.

Pode-se observar que esses voos têm pequena contribuição para a exposição global de passageiros a fontes de radiação natural, que tem uma média mundial de 2,4 mSv/a. Apesar de não ser submetido à regulação, já que esta exposição é vista como voluntária, exposições de voos individuais foram inferiores ao nível de isenção de $10 \mu \mathrm{Sv}$ [6].

Passageiros frequentes, no entanto, podem ter doses superiores a este nível, embora abaixo do nível de intervenção de $10 \mathrm{mSv}$ por ano no Regulamento Brasileiro [6]. Além disso, doses em excesso ao limite de dose para o público a partir de exposições planejadas, de $1 \mathrm{mSv}$ por ano [6], não são esperadas para os passageiros frequentes que fazem voos semanais em qualquer rota doméstica no Brasil. 
Tabela 1. Resumo dos resultados de exposição do público em voos comerciais domésticos, de acordo com o Estado de origem dos voos.

\begin{tabular}{llrrrr}
\hline Estado & Região & Voos/ano & passageiros/ano & $\begin{array}{r}\text { Dose coletiva } \\
\text { (pessoa.Sv/a) }\end{array}$ & $\begin{array}{l}\text { Dose média } \\
(\boldsymbol{\mu} \text { Sv / vô) }\end{array}$ \\
\hline DF & CO & 80.080 & 12.091 .456 & 45,5 & 3,76 \\
GO & CO & 18.356 & 2.130 .856 & 5,2 & 2,44 \\
MS & CO & 10.764 & 1.337 .492 & 3,63 & 2,71 \\
MT & CO & 21.060 & 2.205 .632 & 6,25 & 2,83 \\
AC & N & 3.016 & 394.368 & 1,51 & 3,83 \\
AM & N & 17.784 & 2.437 .916 & 11,75 & 4,82 \\
AP & N & 2.132 & 341.224 & 0,38 & 1,11 \\
PA & N & 27.664 & 3.422 .484 & 10,63 & 3,11 \\
RO & N & 8.684 & 972.712 & 3,1 & 3,19 \\
RR & N & 1.456 & 237.328 & 0,51 & 2,14 \\
TO & N & 5096 & 478.452 & 1,15 & 2,41 \\
AL & NE & 5.565 & 872.185 & 4,21 & 4,83 \\
BA & NE & 53.872 & 7.809 .672 & 28,09 & 3,6 \\
CE & NE & 23.504 & 3.673 .748 & 16,21 & 4,41 \\
MA & NE & 11.336 & 1.728 .688 & 6,15 & 3,56 \\
PB & NE & 6.032 & 959.192 & 5,32 & 5,54 \\
PE & NE & 32.448 & 4.919 .356 & 22,37 & 4,55 \\
PI & NE & 2.758 & 916.240 & 3,3 & 3,6 \\
RN & NE & 11.180 & 1.819 .220 & 8,1 & 4,45 \\
SE & NE & 7852 & 1.046 .344 & 3,44 & 3,29 \\
PR & S & 54.860 & 7.423 .000 & 16,69 & 2,25 \\
RS & S & 43.004 & 5.812 .924 & 19,03 & 3,27 \\
SC & S & 30.992 & 4.113 .668 & 8,95 & 2,18 \\
ES & SE & 19.084 & 2.428 .660 & 5,62 & 2,32 \\
MG & SE & 80.497 & 9.373 .561 & 23,64 & 2,52 \\
RJ & SE & 114.296 & 16.877 .900 & 51,23 & 3,04 \\
SP & SE & 236.496 & 33.209 .540 & 108,52 & 3,27 \\
\hline
\end{tabular}

CO: Centro-Oeste; N: Norte; NE: Nordeste; S: Sul; SE: Sudeste. 
Observa-se que as maiores doses são relacionadas a voos da região Nordeste e ao estado do Amazonas, por terem grande número de voos mais longos, em direção aos estados das demais regiões do país, em particular para São Paulo, Brasília e Rio de Janeiro. Outros estados da região Norte apresentam valores menores de dose média por voo por terem grande número de voos regionais. O grande número de voos entre Rio, São Paulo e Brasília, por envolverem distancias relativamente curtas, levam a uma menor dose média por voo relacionados a estes estados e ao Distrito Federal.

A dose coletiva total a membros da tripulação foi estimada em cerca de 22,4 pessoa.Sv/a. Considerando-se o limite ocupacional de $850 \mathrm{~h}$ de voo por ano para tripulantes e números de voos de cada rota, a dose ocupacional individual média seria de cerca de $0,76 \mathrm{mSv} / \mathrm{a}$.

Considerando tanto o número máximo de voos por rota por ano e o limite legal de 850 horas de voo para qualquer tripulante $[7,8]$, cerca de $15 \%$ dos voos pode levar a doses individuais para tripulantes acima do limite anual para os membros do público de $1 \mathrm{mSv} / \mathrm{a}$, considerando que um único grupo está trabalhando em cada voo. A dose máxima individual a um membro da tripulação, considerando os regulamentos sobre período mínimo de descanso semanal [8], seria então inferior a $2 \mathrm{mSv} / \mathrm{a}$.

\section{CONCLUSÃO}

As doses individuais em voos domésticos comerciais no Brasil em 2011 estão na faixa de 0,03 $8,8 \mu \mathrm{Sv}$ por voo, dependendo da rota e do tipo de avião. As doses decorrentes dos voos mais frequentes no país estariam em torno de 1,8 $\mu \mathrm{Sv}$, para a rota Rio de Janeiro - São Paulo, e cerca de 3,3 $\mu \mathrm{Sv}$ para a rota São Paulo - Brasília. Estas doses são pequenas, em relação à exposição individual média à radiação cósmica estimada para o Brasil de $0,37 \mathrm{mSv} / \mathrm{a}(370 \mu \mathrm{Sv} / \mathrm{a})$, com uma taxa de variação de 0,24 a 0,76 mSv/a [9].

Doses em excesso ao limite de dose para os membros do público podem ser recebidos por alguns viajantes frequentes e alguns tripulantes. Todas as doses individuais previstas, considerando 
abordagens muito conservativas, são, no entanto, bem abaixo dos níveis de intervenção da legislação brasileira [6].

As doses coletivas estimadas devido a voos comerciais domésticos no Brasil seriam de cerca de 421 pessoa.Sv/a e 22 pessoa.Sv/a para passageiros e para a tripulação, respectivamente. Estes valores representam um acréscimo pequeno à exposição total devido à radiação cósmica no Brasil, estimada em 71.000 pessoa.Sv/a [9].

\section{REFERENCIAS}

[1] UNSCEAR - United Nations Scientific Committee on the Effects of Atomic Radiation, UNSCEAR 2008 Report to the General Assembly, with scientific annexes, Vol.I, Annex B, United Nations, New York (2010).

[2] FAA - Federal Aviation Administration. CARI6: Radiobiology Research Team, http://www.faa.gov/data_research/research/med_humanfacs/aeromedical/radiobiology/cari6/ (2011).

[3] PAN ROTA, em http://www.panrotas.com.br (2011).

[4] ALVES, V.A., ROCHEDO, E.R.R., PRADO, N.M.P.D., WASSERMAN, M.A.V., Assessing public exposure in commercial flights in Brazil, Proceedings of the International Conference on Environmental Radioactivity, Barcelona (2014).

[5] BOEING. 737 operations manual, The Boeing Company (2002).

[6] CNEN - Comissão Nacional de Energia Nuclear. Norma NN 3.01 - Diretrizes Básicas de Radioproteção,em: http://www.cnen.gov.br/ segurança/normas/pdf/Nrm301.pdf (2014).

[7] GOVERnO FEDERAL. Casa Civil, Presidência da República, Lei N. 7183 de 5 de abril de 1984 (1984).

[8] TAM, Manual Geral de Operação, Revisão 5, TAM, São Paulo (2011). 
[9] SALLES, K.C.S. Contribuição para o mapeamento da radioatividade natural no Brasil. Dissertação de Mestrado, Instituto Militar de Engenharia (2015). 